\title{
Four decades of pulmonary tuberculosis in deceased South African miners: trends and determinants
}

\author{
Ntombizodwa Ndlovu, ${ }^{1}$ Eustasius Musenge, ${ }^{1}$ Sung Kyun Park, ${ }^{2}$ \\ Brendan Girdler-Brown, ${ }^{3}$ Guy Richards, ${ }^{4}$ Jill Murray ${ }^{1,5}$
}

\begin{abstract}
- Additional material is published online only. To view please visit the journal online (http://dx.doi.org/10.1136/ oemed-2017-104806).

${ }^{1}$ School of Public Health, Faculty of Health Sciences, University of the Witwatersrand, Johannesburg, South Africa ${ }^{2}$ Department of Epidemiology, School of Public Health, University of Michigan, Ann Arbor, Michigan, USA ${ }^{3}$ School of Health Systems and Public Health, University of Pretoria, Pretoria, South Africa ${ }^{4}$ Division of Critical Care, Charlotte Maxeke Hospital and Faculty of Health Sciences, University of Witwatersrand, Johannesburg, South Africa ${ }^{5}$ National Institute for Occupational Health, National Health Laboratory Service, Johannesburg, South Africa
\end{abstract}

\section{Correspondence to} Witwatersrand, Johannesburg 2193, South Africa; zodwa.ndlovu@wits.ac.za

Received 10 October 2017 Revised 17 April 2018

Accepted 25 May 2018 Published Online First 22 June 2018 Ntombizodwa Ndlovu, School of Public Health, University of the

\section{ABSTRACT \\ Objective We investigated trends and determinants of pulmonary tuberculosis (PTB) in deceased South African miners.}

\section{Methods Statutory autopsies are performed on} miners for occupational lung disease compensation, irrespective of cause of death. Data were extracted from the PATHAUT (Pathology Automation System) autopsy database. PTB trends were analysed and explanatory variables (year of autopsy, age at death, gold employment duration, silicosis and HIV) were evaluated using binary logistic regression modelling. Analyses were stratified by population group because of racial differences in socioeconomic status, employment patterns and access to facilities for autopsies. The analyses were segmented to represent the pre-HIV (1975-1989), rapid HIV spread (1990-2004) and antiretroviral therapy (2005-2014) periods.

Results The proportions of men with PTB at autopsy increased from $4.62 \%$ in 1975 to $27.18 \%$ in 2014 in black miners, and from $2.07 \%$ to $5.19 \%$ in white miners, with peaks in 2007 (43.12\% and 9.51\%, respectively). The magnitude and significance of adjusted ORs of determinants differed by population group and calendar period. PTB was largely associated with silicosis, increasing gold employment duration and year of autopsy (a surrogate for unmeasured confounders, such as unknown HIV status and tuberculosis transmission).

Conclusions Changes in PTB time trends and determinants reflect the complex social and political environment in which mining occurs. Silica dust reduction remains a key intervention for tuberculosis reduction, together with tuberculosis and HIV treatment and management. The autopsy data provide reliable information to monitor progress towards the achievement of industry and national targets to reduce tuberculosis

\section{INTRODUCTION}

SLinked

- http://dx.doi.org/10.1136/ oemed-2018-105315

- http://dx.doi.org/10.1136/ oemed-2018-105318

Check for updates

To cite: Ndlovu $\mathrm{N}$ Musenge E, Park SK, et al. Occup Environ Med 2018:75:767-775
Mining activity is associated with increased tuberculosis (TB) incidence, prevalence and mortality in sub-Saharan Africa. ${ }^{1}$ In studies of active and former South African miners conducted in the period 1991-2001, the prevalence of active and inactive TB based on self-reported history, chest radiography or bacteriology (sputum smear and culture) was between $19.4 \%$ and $35.2 \% .^{2-5}$ None of these studies used autopsy diagnosis or reported time trends. Using similar modalities of diagnosis in cohorts of gold miners studied from 1990 to 2004 the observed annual incidence rates ranged from
Key messages

What is already known about this subject?

- There is a triple epidemic of tuberculosis, silicosis and, in more recent decades, HIV infection in South African miners, but data to monitor tuberculosis trends are limited.

What are the new findings?

- This is the first study to show autopsydiagnosed pulmonary tuberculosis (PTB) trends in both black and white South African miners spanning the pre-HIV (1975-1989), rapid HIV spread (1990-2004) and antiretroviral therapy roll-out (2005-2014) periods.

- Overall, PTB was five times higher in black compared with white miners and increased rapidly in the era of HIV to peak in 2007 in both population groups.

- Silicosis and duration of employment were important determinants of PTB, even in the era of HIV.

How might this impact on policy or clinical practice in the foreseeable future?

- Silica dust control remains key to tuberculosis control in the mining industry.

- Interventions to improve tuberculosis and HIV treatment and management are also important.

806 to 3821 cases per 100000 person years, ${ }^{267}$ and were much higher than national estimates, which increased from 250 per 100000 in $1996^{\circ}$ to 781 per 100000 in $2004 .^{9}$

The South African mining industry continues to rely heavily on migrant workers from rural areas and neighbouring countries. Oscillating migration of mine workers has created a unique social context which contributes to the disruption of TB detection and care, treatment failure, poor treatment outcomes, development of drug-resistant strains and transmission throughout the southern African region. ${ }^{10} 11$ This is exacerbated by overcrowded living conditions and work in dusty, poorly ventilated mine shafts. The Southern African Development Community committed itself to address TB and associated risk factors in miners and affected communities, ${ }^{12}$ and The Global Fund to Fight AIDS, Tuberculosis and Malaria is supporting a coordinated regional response to reduce mining-related TB. ${ }^{13}$ 
Occupational exposures to crystalline silica have resulted in high silicosis prevalence among current $(8.3 \%-19.9 \%)^{14}$ and former $(22.0 \%-36.0 \%)^{15} 16$ South African gold miners. Both silica dust and silicosis are risk factors for pulmonary tuberculosis (PTB). The risk is three times higher in miners with silicosis compared with those without, increases with silicosis severity and is lifelong, continuing after dust exposure cessation. ${ }^{16} 17$

Family separation, another consequence of migrancy, is associated with higher risk sexual practices and increased HIV incidence. ${ }^{11}$ Since its advent in the 1980 s, HIV prevalence in miners has increased to about $30 \%{ }^{18}$ In gold miners, HIV infection has been shown to increase the risk of TB fivefold. ${ }^{6}$ One study has demonstrated a multiplicative, 15-fold increase in PTB risk in silicotic, HIV-positive miners, compared with non-silicotic, HIV-negative miners. ${ }^{19}$

Under the apartheid regime, health services were fragmented along racial lines, with inferior services offered to the black population. Since 1994, the postapartheid government has aligned South Africa's TB control policies with those of the WHO. ${ }^{20}$ Initiatives have included implementation of the Directly Observed Treatment, Short-course (DOTS) strategy from 1995, standardised reporting and improvements in TB control. ${ }^{20}$ Interventions to reduce silica dust exposures and eliminate silicosis, ${ }^{21}$ and to improve the prevention, detection and treatment of HIV and $\mathrm{TB},{ }^{22}$ are in progress. Currently, many mines provide TB services for miners and have $\mathrm{TB}$ cure rates that meet or exceed the WHO target of $85 \% .^{23}$ Even so, the TB epidemic continues.

Strengthening the reporting and surveillance of HIV, TB and silicosis is an essential component of TB control. The main sources of national TB statistics are the National Department of Health's Electronic TB Register, the National Health Laboratory Service's laboratory-based Corporate Data Warehouse, and Statistics South Africa's mortality statistics. Most of these sources provide recent data (from the 1900s onwards) and do not provide industry-specific TB data.

South African law recognises TB in miners as a compensable occupational lung disease, irrespective of HIV status. The law allows for examinations of the cardiorespiratory organs of deceased miners, regardless of the clinical cause of death and with consent from a near relative. ${ }^{24}$ Cardiorespiratory organs are removed locally where the miner dies and preserved in formalin. The organs are submitted to the National Institute for Occupational Health (NIOH) in Johannesburg, where detailed autopsy examinations are performed. Since 1975, the pathological findings, demographic information and work histories have been captured on the PATHAUT (Pathology Automation System) database, which was developed primarily for compensation purposes but is also used extensively for research and surveillance.

From 1975 to 1991, the proportions of black gold miners with autopsy-diagnosed PTB and silicosis increased from $0.9 \%$ to $3.9 \%$ and from $9.3 \%$ to $12.8 \%$, respectively. ${ }^{25}$ These changes were explained by increasing age at death and duration of employment over time. Silicosis and year of autopsy were also significant determinants of PTB. A more recent study demonstrated that the proportion of silicosis which was six times lower in black miners (0.03) than white miners $(0.18)$ in 1975 was 1.5 times higher ( 0.32 vs 0.22 , respectively) by $2007 .{ }^{26}$ The main determinants of silicosis were increasing age and duration of employment in both population groups, and year of autopsy in black miners. These silicosis trend analyses ${ }^{25} 26$ were restricted to men who died of external (unnatural) causes to reduce potential bias arising from increased TB-associated mortality in miners with silicosis.
Our study investigated autopsy-diagnosed PTB trends and determinants in black and white South African miners over a 40-year period (1975-2014). Unlike the previous trend analyses, ${ }^{25} 26$ we included all autopsies regardless of the cause of death, that is, both external and non-external (natural) causes, and all commodity groups, not only gold. The data were analysed by population group because of racial differences in the types and duration of mining employment, intensity of dust exposure, living conditions and access to autopsy examination. Generally, black miners are employed in occupations such as stoping and drilling, which have higher silica dust exposures than the supervisory and maintenance positions held by white miners. Historically, black miners worked short-term contracts, while white miners worked as career miners. Policy changes in the last three decades have allowed black miners to work long-term contracts. This has resulted in increased ages, lengths of employment, cumulative silica exposures and silicosis prevalence. ${ }^{26}$ These factors, together with overcrowded living conditions in hostels or shacks and high HIV prevalence, have increased TB risk in black miners. Furthermore, there is inadequate provision of autopsy services in the rural areas to which many black miners return after leaving the industry. ${ }^{27}$

\section{METHODS}

\section{Study population}

The study population comprised male black and white miners whose cardiorespiratory organs were examined at the $\mathrm{NIOH}$ from 1975 to 2014.

\section{Exposure characteristics}

In the absence of dust measurements, cumulative years of service in the gold mining industry (gold employment duration) were used as proxy measures for cumulative silica dust exposure.

Except where stated otherwise, miners were categorised according to the mining commodity in which they worked for the longest time. Commodity refers to the type of mineral mined.

\section{Disease diagnosis and definitions}

Examinations of the cardiorespiratory organs are performed by experienced pathologists using macroscopic and microscopic procedures, which did not change over the study period.

The pathological diagnosis of PTB was based on the presence of microscopic epithelioid granulomas associated with caseous necrosis in the lungs or regional glands, after exclusion of other causes. Silicosis was defined as the presence of palpable silicotic nodules on macroscopic examination of the lung, confirmed by the presence of microscopic silicotic islets histologically.

Clinical causes of death were obtained from death certificates and other documents submitted to the NIOH. We defined HIV in cases where clinicians and/or $\mathrm{NIOH}$ pathologists stated HIV or any of the following AIDS-related diseases as a cause of death: Kaposi sarcoma, cryptococcal pneumonitis, Pneumocystis jiroveci pneumonia, cytomegalovirus pneumonitis or cryptococcal meningitis. Despite TB being an AIDS-related disease, it was excluded from the definition of HIV because it was the outcome of interest.

\section{Statistical analyses}

Data management and analyses were performed using SAS V.9.3 and Stata V.14 software. Disease proportions are expressed as percentages. Student's t-test and the $\chi^{2}$ test were used to determine differences between means and frequencies, respectively. Linear trends were determined using the Cochrane-Armitage 
trend test for proportions and linear regression for continuous variables. Statistical significance was defined at $p<0.05$.

The analyses were segmented according to phases of the HIV epidemic. Following the first reported cases of HIV/AIDS in South Africa in 1982, ${ }^{28}$ antenatal surveys showed an explosive spread of HIV from $0.8 \%$ in 1990 to approximately $29.0 \%$ in $2007 .{ }^{29}$ In 2004, the national roll-out of antiretroviral therapy (ART) was initiated. ${ }^{30}$ Our analyses reflect the pre-HIV and early HIV (1975-1989), rapid HIV spread (1990-2004), and ART (2005-2014) periods.

Binary logistic regression models were used to estimate the association of PTB $(0=$ absent, $1=$ present $)$ with explanatory variables. The initial selection of explanatory variables was based on a priori knowledge. Variables with crude ORs of $\mathrm{p}<0.25$ in the univariable logistic analysis were included in the full models for black miners. The same variables were included in the models for white miners to allow for comparison and because the distribution of significant variables and categories within variables differed across calendar periods. In the initial models, we included silicosis severity categorised as none, occasional, few, moderate and large numbers of nodules. In both population groups, the adjusted ORs (AORs) were significant and were highest in the group with moderate silicosis and lowest in those with marked silicosis. In the absence of a clear trend between PTB and silicosis severity, silicosis was included as a binary variable in the final models. The explanatory variables in the initial multivariable models were population group (black or white), year of autopsy, age at death, gold mining duration, silicosis $(0=$ absent, $1=$ present $)$ and HIV $(0=$ absent, $1=$ present). The final models were stratified by population group because of effect modification and were segmented as explained above. HIV was excluded from the 1975-1989 models because the first case of HIV in our study population was recorded in 1987 and there were few cases of HIV $(n=4)$ before 1990 .

Interaction terms for year and age, year and duration, age and duration, and silicosis and HIV were included in the initial models. Although some of the interaction terms were statistically significant, none were included in the final models because little interaction was demonstrated in interaction plots and their inclusion made little difference to the AORs. In the interests of parsimony, these interaction terms were omitted from the models.

Postregression analyses were performed using the Hosmer-Lemeshow goodness-of-fit test. This test was selected rather than Pearson's goodness-of-fit test because more than $25 \%$ of the expected cells contained fewer than five cases. In this test, $\mathrm{p}$ values of $<0.05$ indicate poor fit, that is, that the observed and expected numbers of PTB cases and non-cases differ more than expected by chance.

To determine if excluded cases biased our findings, we used multiple iterative chained equations to create a data set containing 10 imputations of missing values for age and employment duration for black miners. The mechanism of missingness was defined as missing at random. The final logistic regression models were applied to the imputed data set.

\section{RESULTS}

\section{Study population}

The study population consisted of 83972 deceased miners after exclusion of groups with few cases (female miners and population groups other than black or white) and cases with missing age or employment information (table 1).
Table 1 Numbers of cases on the PATHAUT (Pathology Automation System) database and reasons for exclusions

\begin{tabular}{lcc}
\hline Exclusion criteria & Excluded & Remaining \\
\hline All cases (1975-2014) & 369 & 101167 \\
Female* $^{*}$ & 1936 & 109798 \\
Race not black or white & 3937 & 103925 \\
No age at death or invalid age (<16 or $>$ 100 years) & 2506 & 101419 \\
Commodity not stated & 17447 & 83972 \\
Employment duration, 0 or missing & 26195 & \\
\hline Total & & \\
\hline
\end{tabular}

*Data available from 2005.

There were 53248 (63.41\%) autopsies in black and 30724 $(36.59 \%)$ in white miners (table 2). Black miners were younger and had significantly shorter periods of employment. Most blacks were active miners (79.20\%), whereas most whites were former (86.93\%) miners. The majority were gold miners (77.98\%). The crude proportion of silicosis in black miners was 0.62 that of white miners, and the crude proportion of HIV was 48.96 times higher in black miners.

There were 7389 cases of HIV. Of these, 4705 were defined using clinical causes of death and 2684 cases using a pathological cause of death. Of the HIV-negative cases, 5837 had neither a clinical nor a pathological cause of death stated.

\section{Pulmonary tuberculosis}

Autopsy-diagnosed PTB was detected in 9980 (11.88\%) miners and was five times higher in black miners compared with white miners (table 2). The proportions of PTB by calendar period and population group are provided in online supplementary table S1. The proportions of PTB in black and white miners with autopsy-diagnosed silicosis were $31.34 \%(\mathrm{n}=2233)$ and $6.44 \%$

Table 2 Characteristics of the study population, by population group (1975-2014)

\begin{tabular}{|c|c|c|c|}
\hline Characteristics & $\begin{array}{l}\text { Black } \\
n=53248\end{array}$ & $\begin{array}{l}\text { White } \\
n=30724\end{array}$ & $P$ values \\
\hline Age (years), mean $\pm S D$ & $39.73 \pm 11.27$ & $61.70 \pm 13.98$ & $<0.0001$ \\
\hline $\begin{array}{l}\text { Employment duration } \\
\text { (years), mean } \pm S D\end{array}$ & $10.51 \pm 8.11$ & $24.95 \pm 8.90$ & $<0.0001$ \\
\hline \multicolumn{4}{|c|}{ Employment status* ${ }^{*}$ (\%) } \\
\hline Current miner & $5385(79.20)$ & $473(13.07)$ & $<0.0001$ \\
\hline Former miner & $1414(20.80)$ & $3147(86.93)$ & \\
\hline \multicolumn{4}{|l|}{ Commodity†, n (\%) } \\
\hline Gold & 41308 (77.58) & $24170(78.67)$ & $<0.0001$ \\
\hline Platinum & $6369(11.96)$ & $1087(3.54)$ & \\
\hline Coal & $3414(6.41)$ & $1945(6.33)$ & \\
\hline Asbestos & $1178(2.21)$ & $741(2.41)$ & \\
\hline Other $\ddagger$ & $979(1.84)$ & $2781(9.05)$ & \\
\hline Any gold service§ & $43506(81.70)$ & $26203(85.29)$ & \\
\hline Silicosis, n (\%) & $7125(13.38)$ & $6817(22.19)$ & $<0.0001$ \\
\hline HIV, n (\%) & $7302(13.71)$ & $87(0.28)$ & $<0.0001$ \\
\hline $\begin{array}{l}\text { Pulmonary tuberculosis, } \\
\mathrm{n}(\%)\end{array}$ & $8977(16.8)$ & $1003(3.26)$ & $<0.0001$ \\
\hline \multicolumn{4}{|c|}{$\begin{array}{l}\text { *Data available from } 2007 . \\
\text { tCommodity is defined as the mining industry in which miners worked the longest. } \\
\text { fIncludes aluminium, antimony, cement, chrome, construction, environmental } \\
\text { asbestos, Eskom, fluorspar, phosphate, industry, lead, lime, mica, minerals, nickel, } \\
\text { quarry, refinery, railways, silica, steel, tin, uranium, vanadium, works and zinc. } \\
\text { §Any gold service is defined as employment for at least } 0.1 \text { years in the gold mining } \\
\text { industry. }\end{array}$} \\
\hline
\end{tabular}


Black miners
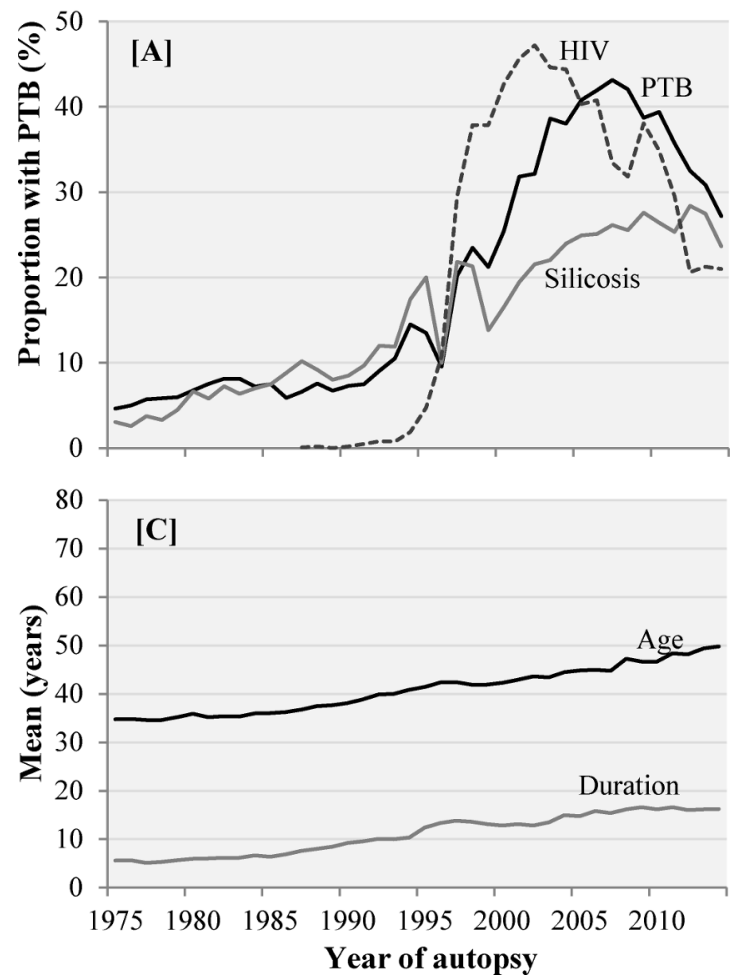

White miners
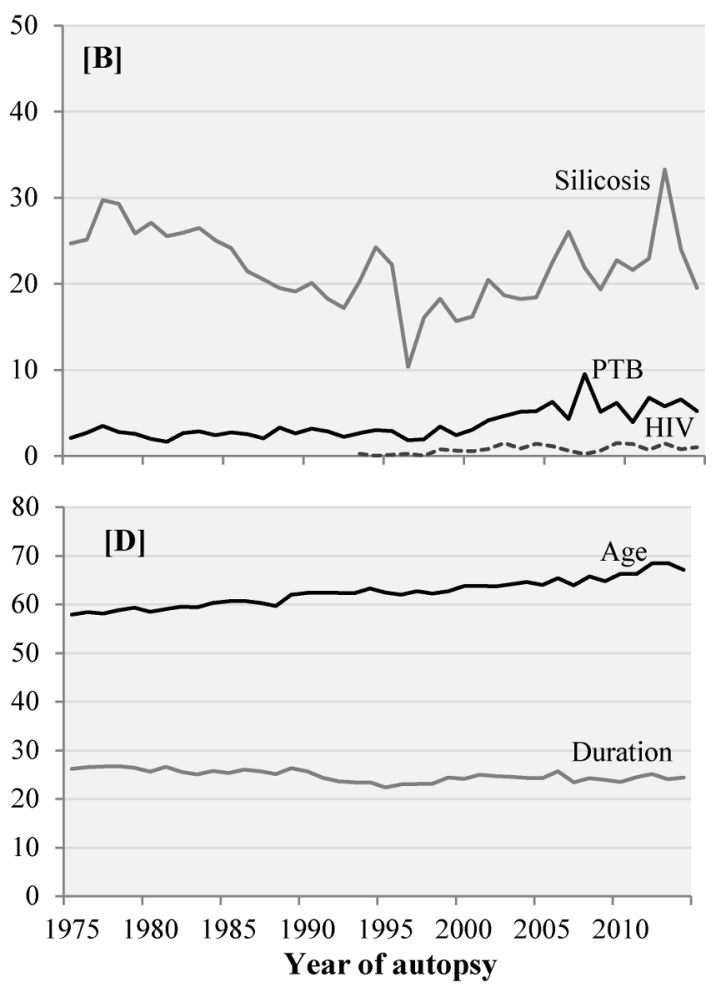

Figure 1 Crude proportions of pulmonary tuberculosis (PTB), silicosis and HIV (A and B), and mean ages and mean employment duration (C and D) in black and white miners from 1975 to 2014.

$(\mathrm{n}=439)$, respectively. Silicosis severity was similar in both population groups and was mild in $1816(67.96 \%)$, moderate in 649 (24.29\%) and marked in 153 (5.73\%) cases.

In black and white miners with HIV, 35.89\% $(n=2621)$ and 10.34\% ( $\mathrm{n}=9$ ) had PTB, respectively. Among those who were HIV-negative, PTB was present in $13.83 \%(n=6356)$ of black and $3.24 \%(n=994)$ of white men.

The proportions of PTB increased across the three calendar periods and the distribution of PTB within each variable differed by population group (see online supplementary table S1).

\section{Trends}

All linear time trends were significant $(\mathrm{p}<0.001)$. Disease trends are shown in figure $1 \mathrm{~A}, \mathrm{~B}$. The lower than expected proportions of PTB and silicosis in 1996 were due to incomplete data entry on the PATHAUT database. In black autopsied miners, PTB increased from $4.62 \%$ in 1975 , peaked at $43.12 \%$ in 2007 and decreased to $27.18 \%$ in 2014 ; silicosis increased from $3.03 \%$ to $23.62 \%$ over the study period; and HIV increased rapidly from below 1.00\% from 1987 to 1993, to a peak in 2002 (47.20\%), and then decreased to $20.96 \%$ in 2014 .

In white autopsied miners, the proportions of PTB fluctuated between $2.00 \%$ and $3.00 \%$ from 1975 to 2000, increased to $9.51 \%$ in 2007 and decreased to $5.19 \%$ in 2014 . The proportions of silicosis declined from $24.88 \%$ in 1975 to $19.51 \%$ in 2014. The proportions of HIV were low (range: $0.12 \%-1.49 \%$ ) and increased over the study period.

Figure 1C,D illustrates increases in the mean ages of deceased miners from $34.71 \pm 11.21$ years in 1975 to $49.75 \pm 11.27$ years in 2014 in black men, and from $57.89 \pm 13.52$ years to $67.05 \pm 12.21$ years in white men. The mean duration of employment (all mining commodities) increased from 5.66 \pm 5.73 years to $17.17 \pm 9.72$ years in black miners, and decreased from $26.16 \pm 11.35$ years to $24.38 \pm 10.77$ years in white miners.

\section{Determinants of PTB trends}

The goodness-of-fit test $p$ values were $p<0.0001$ for the 1990 2004 and 2005-2014 models for black miners, and $p=0.042$ for the 1990-2004 model for white miners. The modelling process was repeated in a $25 \%$ random sample of the study population, and good fit $(p>0.05)$ was obtained for the models in each calendar period.

Tables 3 and 4 present the unadjusted and adjusted ORs for PTB by calendar period in black and white miners, respectively.

\section{5-1989}

In black miners, silicosis, increasing age and year of autopsy group were significant predictors of PTB in the 1975-1989 calendar period. The results for duration of employment are discussed ahead. The odds of PTB in miners with silicosis were three times higher $(\mathrm{AOR}=3.08,95 \% \mathrm{CI} 2.65$ to 3.57$)$ than in non-silicotic miners. Compared with miners younger than 30 years of age, the odds of PTB increased with increasing age, from $\mathrm{AOR}=1.33(95 \% \mathrm{CI} 1.15$ to 1.54$)$ in the age group $30-39$ years to $\mathrm{AOR}=2.47$ (95\% CI 1.92 to 3.18) in those aged 60 years and older. In this calendar period, the AORs of PTB were only significantly elevated for miners who died in 1980-1984 (AOR $=1.31$, 95\% CI 1.15 to 1.49 ) using $1975-$ 1979 as the reference group.

In white miners, silicosis $(\mathrm{AOR}=2.21,95 \% \mathrm{CI} 1.72$ to 2.76$)$ was a significant determinant of PTB. Compared with miners with no gold mining service, the odds of PTB were also significantly elevated in miners with $10-19$ and 20-29 years of 


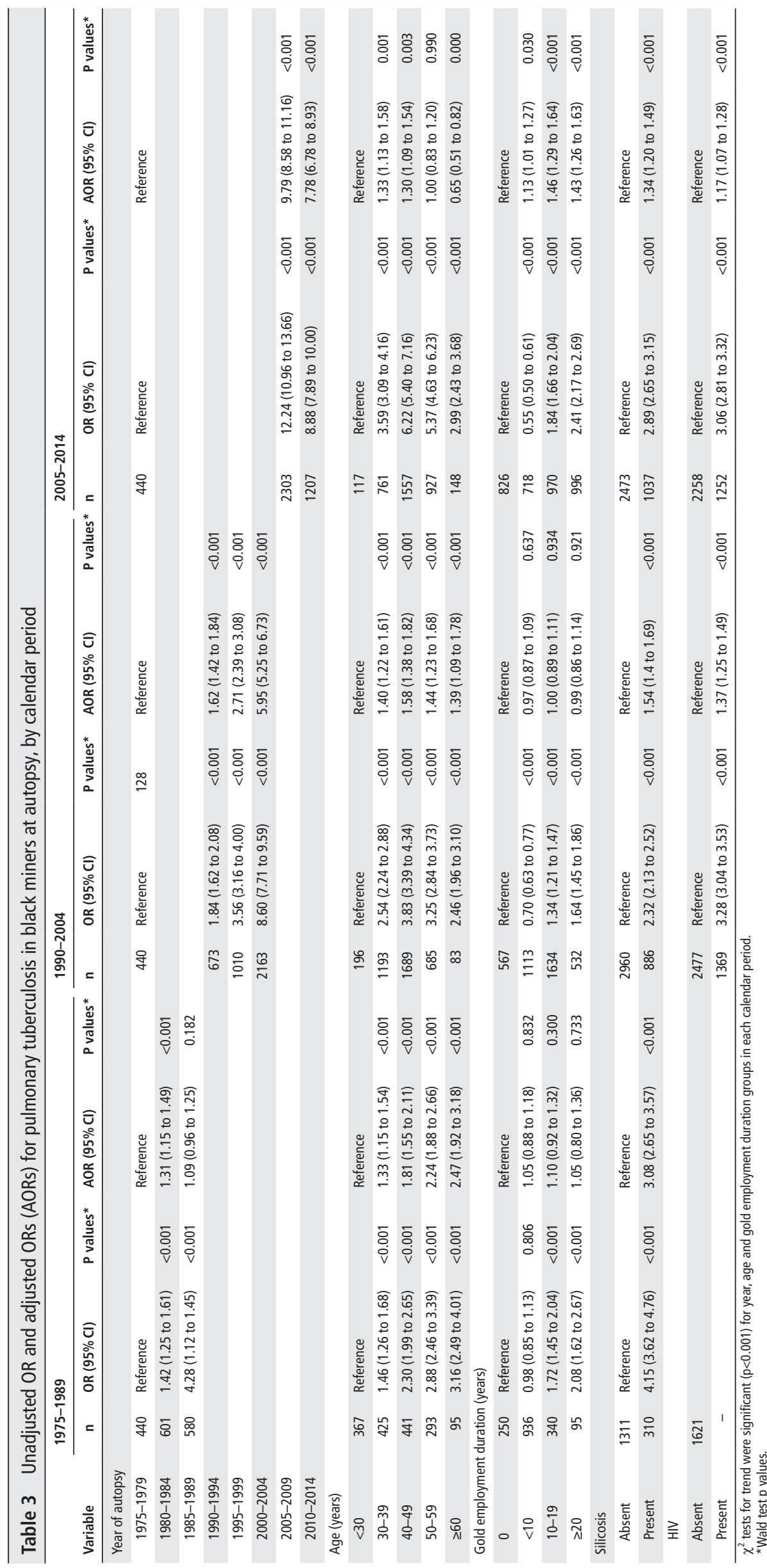




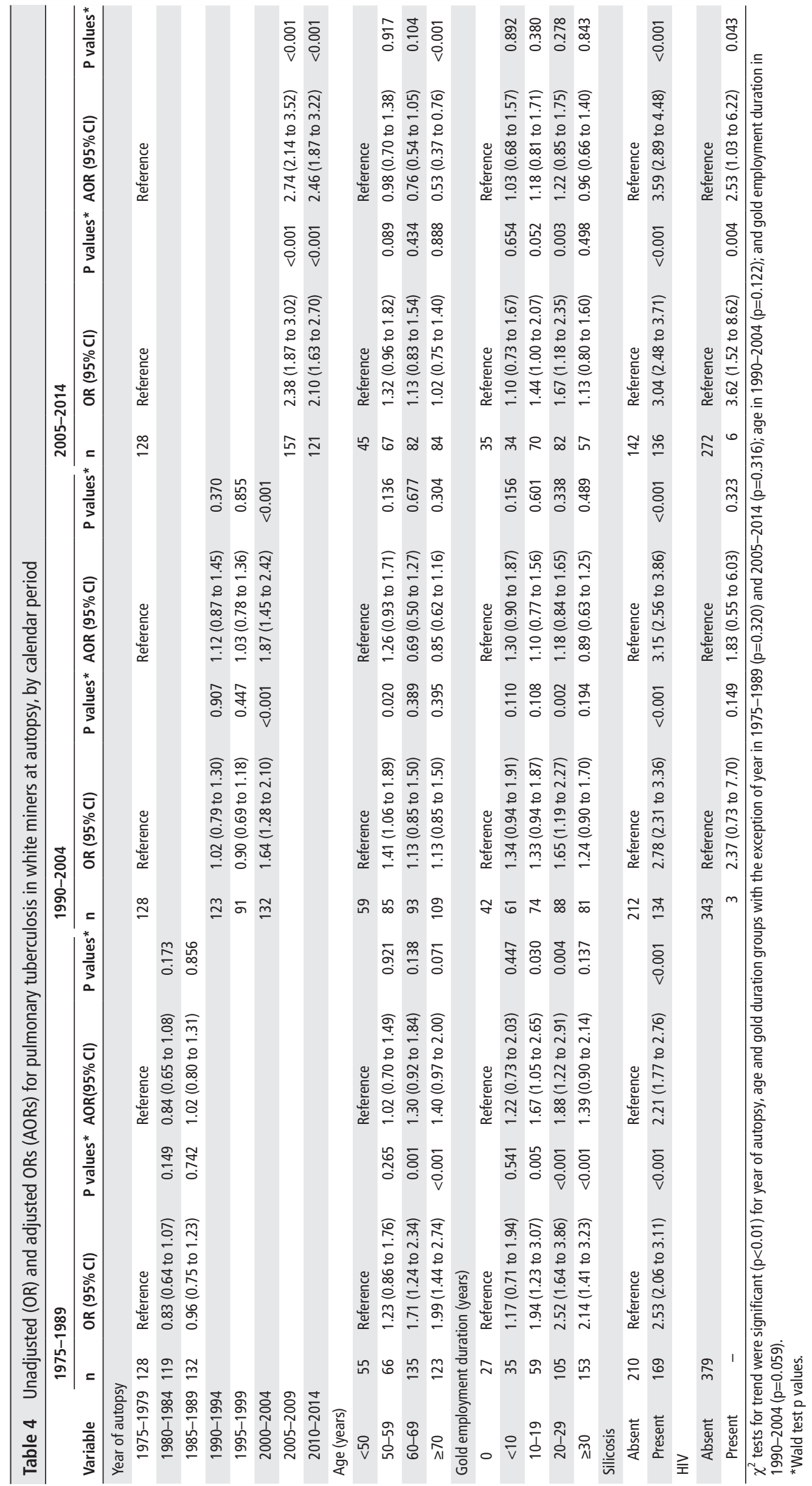


employment in gold mines $(\mathrm{AOR}=1.67,95 \% \mathrm{CI} 1.05$ to 2.65 , and $\mathrm{AOR}=1.88,95 \% \mathrm{CI} 1.22$ to 2.91 , respectively).

\section{0-2004}

In 1990-2004, increasing year of autopsy was the most significant determinant of PTB in black miners. The AORs for silicosis and age were significant but lower than in 1975-1984. The pattern of increasing AORs with increasing age observed in the previous calendar period (1975-1989) was not observed. The AOR for HIV was also significant $(\mathrm{AOR}=1.37,95 \% \mathrm{CI} 1.25$ to 1.49).

In white miners, the AORs for PTB in miners with silicosis were significant and higher than in the previous calendar period. Year of autopsy was significant in 2000-2004.

\section{5-2014}

For black miners, the AORs for PTB continued to increase with increasing year of autopsy and were highest in 2005-2009 $(\mathrm{AOR}=9.79,95 \% \mathrm{CI} 8.58$ to 11.16$)$. The AORs were significantly elevated and increased with increasing gold employment duration. The AORs for PTB were significantly elevated in black miners aged 30-49 years, were not significant in the age group 50-59years and were significantly reduced in those aged 60 years and older $(\mathrm{AOR}=0.65,95 \% \mathrm{CI} 0.51$ to 0.82$)$. The AORs for PTB in miners with silicosis and HIV were significantly elevated but were lower than in the previous calendar period.

In white miners, the AORs for PTB were significantly elevated in miners with silicosis and in each year category, and were higher than in the previous calendar period. The AOR for PTB in the HIV-positive was significantly higher than that in the HIV-negative $(2.53,95 \%$ CI 1.03 to 6.22$)$ and was higher than in black miners (AOR $=1.17,95 \% \mathrm{CI} 1.07$ to 1.28$)$. However, the overlap in CIs indicates that the difference by population group was not statistically significant.

\section{Duration of employment}

To further explore the association between PTB and duration of employment, logistic regression modelling was repeated excluding the silicosis variable (model 2 ). In these models, the AORs for PTB increased with increasing duration (see online supplementary tables S2 and S3) and were significantly elevated in miners with at least 10 years of gold mining employment in 1975-1989 and 2005-2014, but not in 1990-2004.

\section{Missing data}

Of the 26195 excluded cases (table 1), 24229 had missing values: 19727 (75.31\%) were black and 4502 (17.19\%) were white miners. There were 4267 (16.29\%) cases with missing age, 3309 $(12.63 \%)$ with missing commodity and 21898 (83.60\%) with missing employment duration. Among the excluded cases, 3008 (13.77\%) miners had PTB. The AORs and standard errors (SEs) were similar in models using complete cases or imputed data for black miners (see online supplementary table S4).

\section{DISCUSSION}

This study investigated PTB diagnosed pathologically at autopsy in South African miners over four decades of major political, legislative and policy reforms and economic changes in the industry. During this period neither the statutory criteria for access to autopsy compensation nor the pathological criteria for the diagnosis of PTB and silicosis changed. Our main findings were that even before the advent of HIV, the proportions of miners with PTB were high; were five times higher in black compared with white miners; increased over time in both black and white miners; and the magnitude and distribution of determinants of the disease differed by population group and calendar period.

\section{Black miners}

\section{Pre-HIV period}

In 1975-1989, the proportions of PTB in black miners were low but increased over time. During this period, mine dust levels did not change, ${ }^{31}$ silicosis rates in black miners increased ${ }^{26}$ and two important work practice changes were implemented. In 1975, workforce stabilisation, which allowed experienced black miners to work long-term contracts, was introduced, and from 1983 miners who completed TB treatment successfully were permitted to resume underground work. ${ }^{11}$ Prior to this, miners with TB were permanently excluded from working in mines or in dusty surface work. Hence, TB cases in the PATHAUT database prior to 1983 were largely men who had either not been clinically diagnosed or who were awaiting discharge from employment. These policies resulted in an older workforce and increased cumulative dust exposures, which in turn increased silicosis and PTB risks.

In this early period, we could not compare observed PTB trends with those in other South African populations as there are no reliable mortality data. ${ }^{32}$ Silicosis, increasing age and increasing gold mining employment duration were the main PTB determinants in black miners and reflect industry changes at this time.

\section{Rapid HIV spread period}

In 1990-2004, the steep increase in the proportions of black miners with PTB at autopsy coincided with the initiation and rapid spread of $\mathrm{HIV}^{29}$ and HIV-related mortality. ${ }^{33}$ Increasingly, the year of autopsy was the most significant predictor of PTB in black miners. Although statistically significant, the AOR $(\mathrm{AOR}=1.37)$ for HIV was much lower than expected. The definition of HIV that was used is problematic and is discussed ahead. Year of autopsy possibly subsumed the effects of HIV as it is a surrogate for unmeasured confounders. These include the unknown HIV status of many autopsied miners and thus possible misclassification of HIV-negative cases and ongoing and onward transmission of Mycobacterium tuberculosis to both HIV-negative and HIV-positive miners because of high TB prevalence. ${ }^{34}$

\section{ART period}

The peaks in PTB proportions that we observed in 2007 for both population groups occurred around the same time as peak national TB mortality. Pillay-van Wyk et $a l^{32}$ reported combined HIV/AIDS and TB mortality trends based on death notifications from 1997 to 2012. Both the crude and age-adjusted trends increased rapidly from 1997 to peaks in 2006 and declined thereafter. Lim et al, ${ }^{35}$ using data from various sources and autopsies, observed a similar pattern of mortality in platinum miners from 1992 to 2012, with a peak in 2003. The declines in mortality observed after the peaks are associated with the roll-out of ART.

In black miners, year of autopsy continued to be the most significant predictor of PTB in 2005-2014. Age increased the likelihood of PTB in miners aged 30-49 years. However, no association was observed among those aged 50-59 years probably because of a healthy worker effect caused by older miners leaving the industry due to ill-health. The reduced odds of PTB among black miners older than 60 years are explained by selection bias due to poor autopsy uptake by former black miners. ${ }^{27}$ 
Gold mining employment duration was a significant determinant of PTB in black miners in 2005-2014. A dose-related association between increasing cumulative silica dust exposure and PTB risk has been described. ${ }^{17}$ However, in a recent study of copper miners, the association was only significant in the highest exposure group. ${ }^{36}$

\section{White miners}

Few studies have investigated PTB in white South African miners. In a cohort of white gold miners, no PTB deaths were recorded in men who died in the $1970 \mathrm{~s} .{ }^{37}$ In another cohort, 115 of 2255 (5.1\%) miners developed PTB over a 25 -year period (19681971 to 1995$).{ }^{17}$ We observed that the proportions of PTB in white miners remained low well into the period of rapid HIV spread but doubled from 2000 to 2007.

Silicosis was the most important determinant of PTB in white miners throughout the study. The significantly elevated odds of PTB by year group from 2000 to 2004 onwards suggest undisclosed HIV infection in white miners as well as onward transmission of TB to HIV-negative miners. ${ }^{34}$ However, the higher than expected AORs for PTB and HIV in white miners in 2005-2014 $(\mathrm{AOR}=2.53$ vs $\mathrm{AOR}=1.37$ in black miners $)$ may be an anomaly arising from the small numbers of HIV-positive white miners with PTB.

In 2003, voluntary industry milestones were set to reduce $95 \%$ of all respirable crystalline silica dust measurements to below the occupational exposure limit (OEL) of $0.1 \mathrm{mg} / \mathrm{m}^{3}$ and to ensure no new cases of silicosis in novice miners who entered the industry after $2008 .{ }^{21}$ Updated milestones have reduced the OEL to $0.05 \mathrm{mg} / \mathrm{m}^{3}$ and, in line with the latest national TB guidelines, 22 aim to reduce TB incidence to the national rate or below, and improve access to HIV counselling, testing and ART, and health education and communication by $2024 .{ }^{38}$

\section{Strengths and limitations}

A major limitation of this study is that the autopsy data do not represent all deceased South African miners due to inadequate provision of autopsy services for former black miners in labour sending areas. ${ }^{27}$ This was exacerbated by the overall decline in mine employment, which may partially explain the annual decreases in autopsy numbers from the late 1980s. Furthermore, in the era of HIV, increasing numbers of sick miners, many of whom would have had TB, left the mines and had limited access to autopsy services. ${ }^{18}$ Hence, PTB was probably underestimated.

Other limitations are related to the use of routine, administrative data. The PATHAUT database provides information for compensation, which requires limited demographic and exposure information. The unavailability of dust measurements in the database necessitated the use of an imprecise proxy measure, duration of employment, in our analyses. Increasing gold employment duration was an important determinant of PTB. Our models show that silicosis diluted the effect of duration. This highlights the complexity of disentangling the biological effects of these risk factors. The insignificant AORs for PTB and duration in 1990-2004 might be explained by the dramatic spread of the HIV epidemic in this period.

The definition of HIV is an important limitation of this study. Therefore, the AORs for PTB and HIV should be interpreted with caution. HIV status is not required for the determination of compensation for PTB and is often not stated on the death certificate. The exclusion of TB deaths from the definition of HIV and the 5837 cases which were classified as HIV-negative because no cause of death was stated may have resulted in the underestimation of HIV. Although the proportions of HIV in our study were comparable with the $30 \%$ prevalence rates reported in living miners, ${ }^{218}$ we expected a higher proportion of HIV in deceased miners. Misclassification of some HIV negative cases might have also biased the estimates in our models.

A potential limitation was the large number of cases with missing information which were excluded in our analyses. However, the excluded cases did not bias our findings as similar estimates were obtained in models using complete cases or imputed data. Ideally, standardisation of PTB by, for example, age, population group and social class, would have been appropriate. However, there were no appropriate external population data available for standardisation, but we have demonstrated that in more recent years the trends of PTB in this study were comparable with TB trends in the general South African population. ${ }^{32}$

A strength of the study was the diagnosis of PTB based on pathological findings at autopsy, which have higher specificity and sensitivity than clinical diagnoses. ${ }^{39}$ Furthermore, the pathological diagnostic criteria were constant throughout the study period and thereby enabled comparisons over time. In contrast to other South African studies that reported TB trends from the 1990s onwards, the availability of long-term data enabled us to analyse trends beginning well before the era of HIV.

\section{CONCLUSIONS}

Despite its limitations, the PATHAUT database provides the longest standing source of surveillance information for PTB in the South African mining industry and general population. Although PTB in miners has declined since the initiation of ART, the proportions are still high and indicate continued disease transmission. In our analyses, the major determinants of PTB in the most recent calendar period were year of autopsy, long duration of employment in gold mining, silicosis and HIV. Our findings underscore the complexity of the mining environment and provide clear indications of areas of intervention required to achieve current targets to reduce TB and to address its determinants. Silicosis and gold mining duration are important determinants of PTB, even in the era of HIV; therefore silica dust control remains key to $\mathrm{TB}$ control in the mining industry. Interventions to improve TB and HIV treatment and management are also important. The autopsy data provide reliable information for monitoring the outcomes of interventions to reduce $\mathrm{TB}$ in the mining industry.

Contributors NN: substantially contributed to the conception and design of the work; acquisition, analysis and interpretation of data for the work; and drafting the work and revising it critically for important intellectual content. EM: substantially contributed to the analysis and interpretation of data for the work. SKP: substantially contributed to the design of the work; and analysis and interpretation of data for the work. BG-B: substantially contributed to the analysis and interpretation of data for the work. GR: substantially contributed to the conception and interpretation of data for the work. JM: substantially contributed to the conception of the work; and acquisition and interpretation of data for the work. EM, SKP, BG-B, GR, JM: revising the work critically for important intellectual content. NN, EM, SKP, BG-B, GR, JM: final approval of the version to be published. NN, EM, SKP, BG-B, GR, JM: agreed to be accountable for all aspects of the work in ensuring that questions related to the accuracy or integrity of any part of the work are appropriately investigated and resolved.

Funding The project was supported by Award Number D43ES018744 from the US National Institute of Environmental Health Sciences.

Disclaimer The content is solely the responsibility of the authors and does not necessarily represent the official views of the National Institute of Environmental Health Sciences or the National Institutes of Health.

Competing interests None declared.

Patient consent Next of kin consent obtained. 
Ethics approval This study was approved by the University of the Witwatersrand Human Research Ethics Committee (clearance certificate number, M120236) and the University of Michigan Health Sciences and Behavioural Sciences Institutional Review Board (HUM00069012).

Provenance and peer review Not commissioned; externally peer reviewed. (c) Article author(s) (or their employer(s) unless otherwise stated in the text of the article) 2018. All rights reserved. No commercial use is permitted unless otherwise expressly granted.

\section{REFERENCES}

1 Stuckler D, Basu S, McKee M, et al. Mining and risk of tuberculosis in sub-Saharan Africa. Am J Public Health 2011;101:524-30.

2 Park HH, Girdler-Brown BV, Churchyard GJ, et al. Incidence of tuberculosis and HIV and progression of silicosis and lung function impairment among former Basotho gold miners. Am J Ind Med 2009;52:901-8.

3 Steen TW, Gyi KM, White NW, et al. Prevalence of occupational lung disease among Botswana men formerly employed in the South African mining industry. Occup Environ Med 1997:54:19-26.

4 teWaternaude JM, Ehrlich RI, Churchyard GJ, et al. Tuberculosis and silica exposure in South African gold miners. Occup Environ Med 2006;63:187-92.

5 Trapido AS. An analysis of the burden of occupational lung disease in a random sample of former gold mineworkers in the Libode District of the Eastern Cape. University of the Witwatersrand 2000.

6 Churchyard GJ, Kleinschmidt I, Corbett EL, et al. Mycobacterial disease in South African gold miners in the era of HIV infection. Int J Tuberc Lung Dis 1999;3:791-8.

7 Glynn JR, Murray J, Bester A, et al. Effects of duration of HIV infection and secondary tuberculosis transmission on tuberculosis incidence in the South African gold mines. AIDS 2008;22:1859-67.

8 World Health Organization. Global Tuberclosis Control:WHO Report 1998. 1998 http://apps.who.int/iris/bitstream/10665/63835/1/WHO_TB_98.237.pdf?ua=1 (accessed 3 Feb 2018).

9 World Health Organization. Global Tuberculosis Control Surveillance, Planning, Financing:WHO Report 2006. 2006 http://apps.who.int/iris/bitstream/10665/ 144567/1/9241563141_eng.pdf?ua=1 (accessed 3 Feb 2018).

10 Basu S, Stuckler D, Gonsalves G, et al. The production of consumption: addressing the impact of mineral mining on tuberculosis in southern Africa. Global Health 2009;5:11.

11 Rees D, Murray J, Nelson G, et al. Oscillating migration and the epidemics of silicosis, tuberculosis, and HIV infection in South African gold miners. Am J Ind Med 2010:53:398-404.

12 Southern African Development Community. Declaration on Tuberculosis in the Mining Sector. 2012 http://www.stoptb.org/assets/documents/news/Declaration\%20on\% 20Tuberculosis\%20in\%20the\%20Mining\%20Sector2012English.pdf (accessed 4 Feb 2018).

13 Reiprich G, va Zyl A, Govender V, et al. Heath Focus - TB in the mining sector in Southern Africa (TIMS). 2017. https://www.timssa.co.za/Portals/0/Documents/TIMS TOR\%20for\%20EPE\%20Consultancy.pdf (accessed 4 Feb 2018).

14 Churchyard GJ, Ehrlich R, teWaterNaude JM, et al. Silicosis prevalence and exposureresponse relations in South African goldminers. Occup Environ Med 2004;61:811-6.

15 Trapido AS, Mqoqi NP, Williams BG, et al. Prevalence of occupational lung disease in a random sample of former mineworkers, Libode District, Eastern Cape Province, South Africa. Am J Ind Med 1998;34:305-13.

16 Corbett EL, Charalambous S, Moloi VM, et al. Human immunodeficiency virus and the prevalence of undiagnosed tuberculosis in African gold miners. Am J Respir Crit Care Med 2004;170:673-9.
17 Hnizdo E, Murray J. Risk of pulmonary tuberculosis relative to silicosis and exposure to silica dust in South African gold miners. Occup Environ Med 1998;55:496-502.

18 Bateman C. Annually, $1 \%$ of gold miners die--4\% sent home sick. S Afr Med J 2014;104:160-2.

19 Corbett EL, Churchyard GJ, Clayton TC, et al. HIV infection and silicosis: the impact of two potent risk factors on the incidence of mycobacterial disease in South African miners. AIDS 2000;14:2759-68.

20 Abdool Karim SS, Churchyard GJ, Karim QA, et al. HIV infection and tuberculosis in South Africa: an urgent need to escalate the public health response. Lancet 2009;374:921-33.

21 Department of Labour, South Africa. National Programme for the Elimination of Silicosis. http://www.labour.gov.za/DOL/downloads/documents/useful-documents/ occupational-health-and-safety/Useful\%20Document $\% 20-\% 200 \mathrm{HS} \% 20-\%$ 20 National\%20Programme $\% 20$ for $\% 20$ the\%20Elimination\%20of\%20Silicosis.pdf (accessed 4 Feb 2018).

22 Department of Health, South Africa. National Strategic Plan on HIV, STI's and TB, 2017-2022. 2017. https://www.gov.za/sites/default/files/nsp\%20hiv\%20tb\%20sti_a. pdf (accessed 4 Feb 2018)

23 Dharmadhikari A, Smith J, Nardell E, et al. Aspiring to zero tuberculosis deaths among southern Africa's miners: is there a way forward? Int J Health Serv 2013;43:651-64.

24 Republic of South Africa. Occupational Disease in Mines and Works Act No. 78 of 1973. Pretoria: Government Printer, 1973.

25 Murray J, Kielkowski D, Reid P. Occupational disease trends in black South African gold miners. An autopsy-based study. Am I Respir Crit Care Med 1996;153:706-10.

26 Nelson G, Girdler-Brown B, Ndlovu N, et al. Three decades of silicosis: disease trends at autopsy in South African gold miners. Environ Health Perspect 2010;118:421-6.

27 Ndlovu N, Nelson G, Vorajee N, et al. 38 years of autopsy findings in South African mine workers. Am J Ind Med 2016:59:307-14.

28 Ras GJ, Simson IW, Anderson R, et al. Acquired immunodeficiency syndrome. SAMJ 1983:64:140-2.

29 Department of Health, South Africa. The 2011 National Antenatal Sentinel HIV and Syphilis Prevalence Survey in South Africa. 2011 https://www.health-e.org.za/wpcontent/uploads/2013/05/f0980fb5107a7ce543a8bd5730e52333.pdf (4 Feb 2018).

30 Evans D. Ten years on ART - where to now? S Afr Med J 2013;103:229-30.

31 Leon RN SM, Davies AW, Davies JCA. Report of the Commission of Inquiry into Safety and Health in the Mining Industry. Braamfontein 1995.

32 Pillay-van Wyk V, Msemburi W, Laubscher R, et al. Mortality trends and differentials in South Africa from 1997 to 2012: second National Burden of Disease Study. Lancet Glob Health 2016;4:e642-53.

33 Byass P, Kahn K, Fottrell E, et al. Using verbal autopsy to track epidemic dynamics: the case of HIV-related mortality in South Africa. Popul Health Metr 2011;9:46.

34 Sonnenberg P, Glynn JR, Fielding K, et al. HIV and pulmonary tuberculosis: the impact goes beyond those infected with HIV. AIDS 2004;18:657-62.

35 Lim MS, Dowdeswell RJ, Murray J, et al. The impact of HIV, an antiretroviral programme and tuberculosis on mortality in South African platinum miners, 19922010. PLoS One 2012; 7:e38598.

36 Ngosa K, Naidoo RN. The risk of pulmonary tuberculosis in underground copper miners in Zambia exposed to respirable silica: a cross-sectional study. BMC Public Health 2016;16:855.

37 Wyndham CH, Bezuidenhout BN, Greenacre MJ, et al. Mortality of middle aged white South African gold miners. $\mathrm{Br} J$ Ind Med 1986;43:677-84.

38 Msiza D. The road to sero harm: New milestones: Department of Mineral Resourses, 2014 http://www.mhsc.org.za/sites/default/files/Mr\%20Msiza\%202014\% 20Milestones_0.pdf (accessed 4 Feb 2018).

39 Field N, Murray J, Wong ML, et al. Missed opportunities in TB diagnosis: a TB processbased performance review tool to evaluate and improve clinical care. BMC Public Health 2011:11:127. 